\title{
Almost Sure Rotation Number of Circle Endomorphisms
}

\author{
Sylvain Crovisier
}

\begin{abstract}
We introduce the almost sure rotation number $\rho_{\text {as }}$ for some circle endomorphisms $f$. From ergodic theory, this extends the classical rotation number. By restriction to a particular piecewise linear family, we show that $\rho_{\text {as }}$ has Hölder variations with $f$ and is irrational on a full Lebesgue measure set of parameters.
\end{abstract}

\section{Introduction}

One would expect that the theory of dynamical systems ought to be able to describe the asymptotic behaviour of "most orbits" of "most dynamical systems". This is the goal we want to adopt here in order to study circle endomorphisms and their rotation number.

The rotation number was introduced by H. Poincaré for orientation-preserving circle homeomorphisms as a conjugacy invariant. It says how fast orbits rotate around the circle. We consider here a larger class of systems: the circle endomorphisms.

We will denote by $\mathbb{T}^{1}=\mathbb{R} / \mathbb{Z}$ the circle and by $\pi: \mathbb{R} \rightarrow \mathbb{T}^{1}$ its universal covering. A map $f: \mathbb{T}^{1} \rightarrow \mathbb{T}^{1}$ is a circle endomorphism if there exists a continuous map $\tilde{f}: \mathbb{R} \rightarrow \mathbb{R}$ such that $\pi \circ \tilde{f}=f \circ \pi$. It will be convenient to fix arbitrarily a choice for the lift $\tilde{f}$. The set of circle endomorphisms contains orientation preserving circle homeomorphisms but also non-injective maps. A smooth model in this theory, known as the Arnol'd family $\left(\tilde{f}_{a, \omega}\right)$, and parametrized by $a \geq 0$ and $\omega \in \mathbb{R}$, is defined by

$$
\tilde{f}_{a, \omega}(\tilde{x})=\tilde{x}+a \sin (2 \pi \tilde{x})+\omega
$$

for any $\tilde{x} \in \mathbb{R}$. The map $f_{a, \omega}$ is a homeomorphism when $0 \leq a \leq 1 / 2 \pi$.

For any point $x \in \mathbb{T}^{1}$, its rotation number during $n \in \mathbb{N} \backslash\{0\}$ iterations by $\tilde{f}$ is

$$
\rho_{n}(x, \tilde{f})=\frac{\tilde{f}^{n}(\tilde{x})-\tilde{x}}{n},
$$

where $\tilde{x} \in \mathbb{R}$ is any lift of $x$ (i.e. $\pi(\tilde{x})=x$ ). The asymptotic rotation behaviour of $f$ is characterised by taking the limit values of $\rho_{n}(x, \tilde{f})$ when $n$ goes to infinity. A

1991 Mathematics Subject Classification. 37C40, 37E10, 37E45.

Key words and phrases. Expanding circle endomorphism, rotation number, SRB measure.

(C)0000 (copyright holder) 
classical result (see [CGT]) asserts that one gets a compact interval $\mathcal{R}(\tilde{f})$, called the rotation set of $\tilde{f}$ :

$$
\mathcal{R}(\tilde{f})=\bigcup_{x \in \mathbb{T}^{1}} \text { limit points of the sequence }\left(\rho_{n}(x, \tilde{f})\right) .
$$

The rotation set may also be obtained from the measure theory: for any $f$-invariant Borel probability measure $\mu$ over $\mathbb{T}^{1}$, let us define the rotation number of $\mu$ by

$$
\rho(\mu, \tilde{f})=\int_{[0,1)}(\tilde{f}(\tilde{x})-\tilde{x}) \mathrm{d} \mu .
$$

Then,

$$
\mathcal{R}(\tilde{f})=\{\rho(\mu, \tilde{f}), \mu f \text {-invariant probability measure }\}
$$

For some endomorphisms it may happen however that the sequence $\rho_{n}(x, \tilde{f})$ converges for most orbits (i.e. for $x$ in a set with full Lebesgue measure) toward the same number. We call this "physical" rotation number the almost sure rotation number, $\rho_{\text {as }}(\tilde{f})$. We hope $\rho_{\text {as }}(\tilde{f})$ to be defined for most systems $f$ in order to study how it varies. In particular, for any endomorphism $f$, we first introduce the family $\left(f_{\omega}\right)$ after composition by the rotation:

$$
\tilde{f}_{\omega}(\tilde{x})=\tilde{f}(\tilde{x})+\omega .
$$

We then consider, where it is defined, the map

$$
\omega \mapsto \rho_{\text {as }}\left(\tilde{f}_{\omega}\right) \text {. }
$$

Our forthcoming results are motivated by the standard situation of circle diffeomorphisms (Section 2): in this case, $\mathcal{R}(\tilde{f})$ is a singleton, so that $\rho_{\text {as }}(\tilde{f})$ is always defined and called the rotation number of $\tilde{f}$. Its dependence with respect to $\tilde{f}$ is well-understood after Herman's work in [H79, H77]. Figure 1 shows as an example the variations of $\omega \mapsto \rho\left(\tilde{f}_{a, \omega}\right)$ for the Arnol'd family when $a=0.15$.

In order to switch to the non-invertible endomorphisms $f$, one has to require some assumption that guarantees the existence of $\rho_{\text {as }}(\tilde{f})$. This is discussed in Section 3. In the following sections, we restrict our study to a particular 2-parameter family (a piecewise linear model for the Arnol'd endomorphisms), $\left(f_{\lambda, \omega}\right)$, defined as follows, for $\lambda \geq 1$ and $\omega \in \mathbb{R}$ (see Figure 21):

(1) When $\tilde{x} \in\left[0, \frac{1+\lambda}{2 \lambda}\right), \tilde{f}_{\lambda, \omega}(\tilde{x})=\lambda x+\omega$.

(2) When $\tilde{x} \in\left[\frac{1-\lambda}{2 \lambda}, 0\right), \tilde{f}_{\lambda, \omega}(\tilde{x})=-\lambda x+\omega$.

(3) For any $\tilde{x} \in \mathbb{R}, \tilde{f}_{\lambda, \omega}(\tilde{x}+1)=\tilde{f}_{\lambda, \omega}(\tilde{x})+1$.

When $\lambda>3, \rho_{\text {as }}\left(\tilde{f}_{\lambda, \omega}\right)$ is well defined and will be denoted by $\rho_{\text {as }}(\lambda, \omega)$. Figure 3 shows the map $\omega \mapsto \rho_{\text {as }}(\lambda, \omega)$ when $\lambda=3.5$ (compare with Figure 11).

Then, we show in Section 4 that $\rho_{\text {as }}\left(\tilde{f}_{\lambda, \omega}\right)$ varies smoothly:

Theorem 1.1. The maps $\omega \mapsto \rho_{a s}(\lambda, \omega)$ for $\lambda>3$ are Hölder continuous.

In view of Herman's results (Section 2), it is also natural to ask how $\rho_{\text {as }}(\lambda, \omega)$ varies with $\lambda$ and $\omega$. For example, is it mostly rational or irrational? Section 5 deals with this question.

Theorem 1.2. There exists $\lambda_{0}>3$ and a set $E$ with full Lebesgue measure in $\left(\lambda_{0},+\infty\right] \times \mathbb{R}$ such that for any $(\lambda, \omega) \in E, \rho_{a s}(\lambda, \omega)$ is irrational.

Those last two results come from Cr01b. We present here only a sketch of the proofs. A detailed exposition of Theorem 1.1 appeared also in Cr01a. 


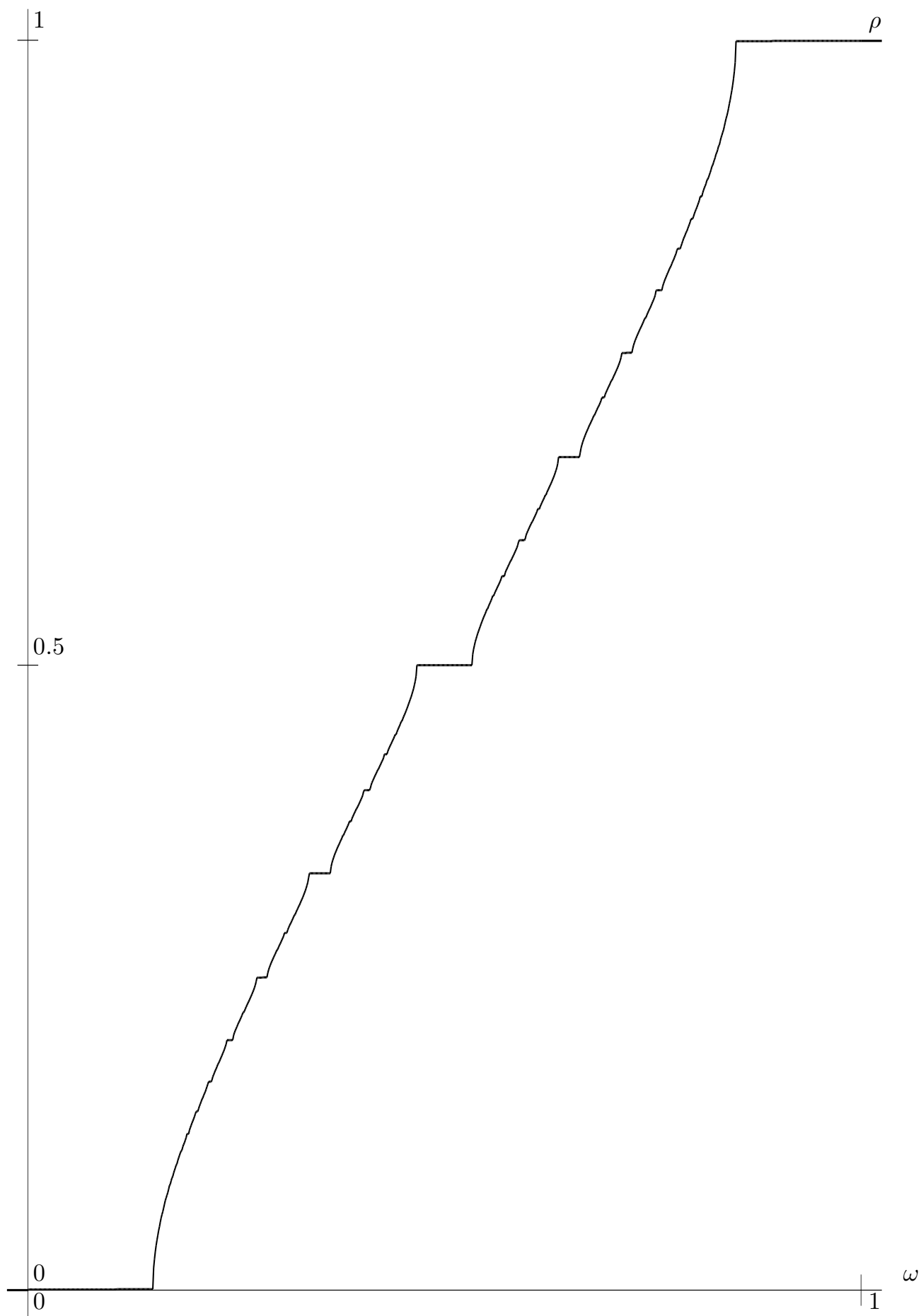

FigURE 1. Rotation number $\rho$ of the Arnol'd diffeomorphisms $\tilde{x} \mapsto$ $\tilde{x}+0.15 \sin (2 \pi \tilde{x})+\omega$. 


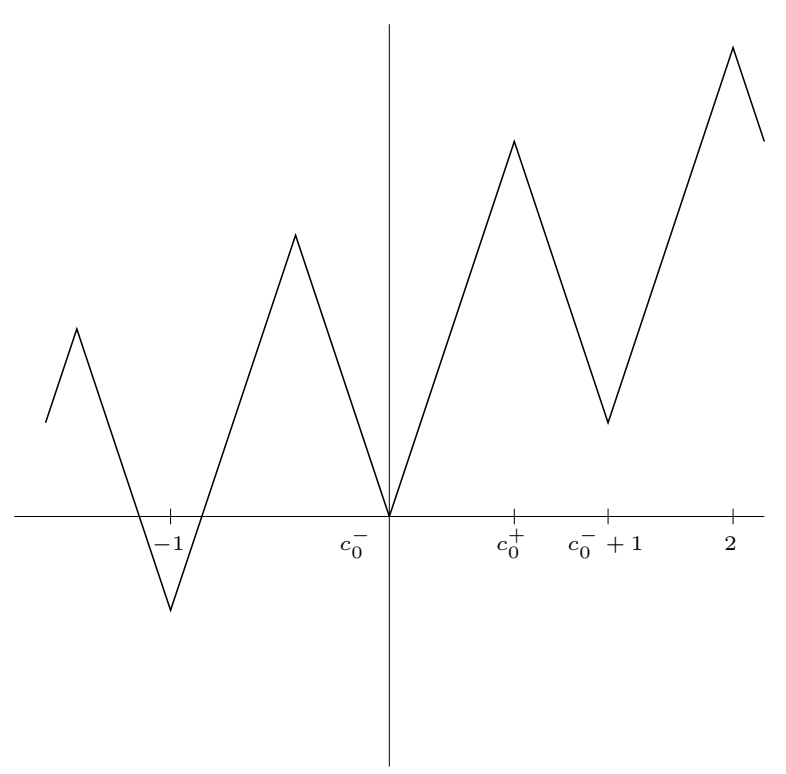

Figure 2. The map $\tilde{x} \mapsto \tilde{f}_{\lambda, \omega}(\tilde{x})$.

\section{The invertible case}

Let us consider an orientation preserving circle homeomorphism $f$ and the associated map $\omega \mapsto \rho(\omega)=\rho\left(\tilde{f}_{\omega}\right)$. V. Arnol'd and M. Herman remarked that its graph is a devil staircase (see [A, H77, H79] and Figure 1):

Proposition 2.1 (Arnol'd, Herman).

(1) The map $\rho$ is continuous and non-decreasing.

(2) For any irrational number $\alpha \in \mathbb{R} \backslash \mathbb{Q}, \rho^{-1}(\alpha)$ is a singleton.

(3) If $f$ satisfies a generic condition $A_{0}$, for any rational number $p / q, \rho^{-1}(p / q)$ has non-empty interior.

Proposition 2.1 says that for a generic map $f$ (in fact, for any $f$ in an open and dense subset of circle orientation preserving homeomorphisms space) $\rho(\tilde{f})$ is rational. On an open and dense subset of $\mathbb{R}, \rho$ takes rational values. On the other hand, Herman proved also:

Theorem 2.2 (Herman). If $f$ is a $C^{3}$-diffeomorphism that satisfies a generic condition $A_{3}$, the map $\rho$ is absolutely continuous and takes irrational values on a non-zero Lebesgue measure set.

The Arnol'd maps with $0<a<1 / 2 \pi$ satisfy both condition $A_{0}$ and $A_{3}$. In the measure sense, rational and irrational values are both relevant. Several improvements to these results have been given later [S88, G93, GS, T92].

\section{The non-invertible case}

3.1. The rotation interval. We will only assume now that $f$ is an endomorphism and for any $\omega \in \mathbb{R}$ the rotation set of $\tilde{f}_{\omega}$ will be denoted by $\mathcal{R}\left(\tilde{f}_{\omega}\right)=$ 


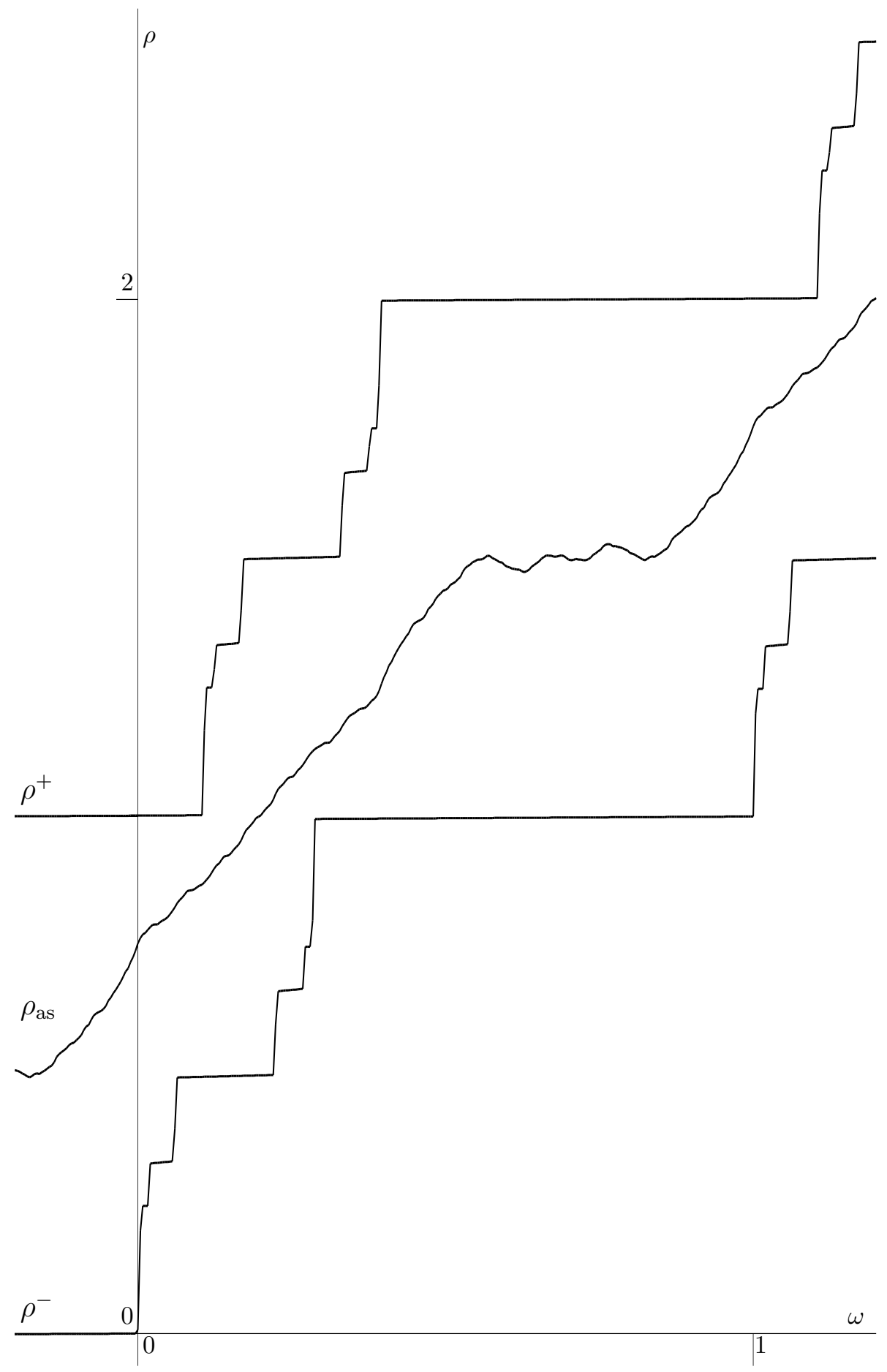

Figure 3 . The maps $\rho^{-}, \rho_{\text {as }}$, and $\rho^{+}$for the slope $\lambda=3.5$. 
$\left[\rho^{-}\left(\tilde{f}_{\omega}\right), \rho^{+}\left(\tilde{f}_{\omega}\right)\right]$. The dependence of $\mathcal{R}\left(\tilde{f}_{\omega}\right)$ in $\omega$ has been studied by P. Boyland in $[\mathbf{B}]$. He showed that essentially Proposition 2.1 still holds for the maps $\omega \mapsto \rho^{-}\left(\tilde{f}_{\omega}\right)$ and $\omega \mapsto \rho^{+}\left(\tilde{f}_{\omega}\right)$. G. Świątek proved however in $\mathbf{S 8 9}$ that Theorem 2.2 fails here:

Theorem 3.1 (G. Świątek). For $f$ in some large class of $C^{2}$ bimodal circle endomorphisms, and $\omega$ in a full Lebesgue measure subset of $\mathbb{R}$, the endpoints $\rho^{-}\left(\tilde{f}_{\omega}\right)$ and $\rho^{+}\left(\tilde{f}_{\omega}\right)$ are both rational.

Here again, Theorem 3.1 applies with the Arnol'd endomorphisms $(a>1 / 2 \pi)$.

3.2. The almost sure rotation number. Those results give a complete topological description of the rotation behaviour of circle endomorphisms. From now on we adopt an ergodic-theoretic viewpoint and consider the almost sure rotation number when it is defined. Note that this is no longer a topological invariant of the dynamics.

The almost sure rotation number is obviously defined when a periodic cycle attracts almost every orbit. The almost sure rotation number is then rational and (at least in the $C^{2}$ topology) this situation is robust. Thus, we will consider the much more interesting endomorphisms $f$ that satisfy the following condition:

There exists a set $\Omega \subset \mathbb{R}$ with positive Lebesgue measure such that

(P) any map $f_{\omega}$ with $\omega \in \Omega$ preserves an ergodic probability measure $\mu_{\omega}$ that is equivalent to the Lebesgue measure on $\mathbb{T}^{1}$.

By Birkhoff's ergodic theorem, for any $\omega \in \Omega$, and Lebesgue almost every point $x \in \mathbb{T}^{1}$, the sequence $\rho_{n}\left(x, \tilde{f}_{\omega}\right)$ converges to $\rho\left(\mu_{\omega}, \tilde{f}_{\omega}\right)$, the almost sure rotation number of $\tilde{f}_{\omega}$.

3.3. Smooth endomorphisms. It is now well-known that the existence of an absolutely continuous invariant measure for families of smooth critical maps is associated to large sets of parameters. This was first proven by M. Jakobson in [J] for the quadratic family but extended later to multimodal maps (see [T93, WY ). This absolutely continuous invariant measure is ergodic and has a positive Lyapounov exponent. By $[\mathbf{L}$, it is thus sufficient to prove then that its support is the full circle in order to get $(\mathrm{P})$. This can be obtained from the hyperbolic behaviour of one-dimensional maps far from the critical points:

Proposition 3.2. There exists in $\left(\frac{1}{2 \pi},+\infty\right)$ a set $A$ of positive Lebesgue measure such that for all $a \in A$, the Arnol'd families $\left(f_{a, \omega}\right)_{\omega \in \mathbb{R}}$ satisfy $(P)$.

This has been proven in Cr01b. The measure is however obtained in an indirect way so that the study of the variations of $\rho_{\text {as }}$ seems difficult.

3.4. Piecewise linear endomorphisms. In order to simplify our study we will now consider piecewise expanding maps: they are known to preserve absolutely continuous probability measures. As a model we will work with the piecewise linear family of bimodal circle endomorphisms $\left(f_{\lambda, \omega}\right)$ as defined in Section 1. Many authors have already been interested in piecewise linear circle maps (see [H79, GS]).

The turning points $c_{0}^{-}=\pi(0)$ and $c_{0}^{+}=\pi(1+\lambda / 2 \lambda)$ will be called the critical points. Property $(\mathrm{P})$ is easily checked: 
Proposition 3.3. For any $\lambda>3$, the family $\left(f_{\lambda, \omega}\right)$ satisfies Property $(P)$ with $\Omega=\mathbb{R}$.

Proof. From $[\mathbf{L Y}, \mathbf{I}], f_{\lambda, \omega}$ preserves at least one absolutely continuous ergodic probability measure $\mu$. Its support is a finite union of disjoint compact intervals where $\mu$ is equivalent to the Lebesgue measure. Let $I$ be one of these intervals. If one assumes that $I$ is not the full circle $\mathbb{T}^{1}$, as $\lambda>3$, the length of $f_{\lambda, \omega}(I)$ is strictly larger than the length of $I$ even if $I$ contains one or the two critical points. This is a contradiction since $I$ should be mapped into itself after some iteration.

For some parameters, it is possible to compute explicitly $\rho_{\text {as }}(\lambda, \omega)$ (see Cr01a) and show that the map $\rho_{\text {as }}$ is not in general an increasing function of $\omega$ (see Figure 3).

\section{Regularity}

4.1. Main results. For any parameter $(\lambda, \omega)$ we will denote by $\mu_{\lambda, \omega}$ the invariant probability measure which is equivalent to the Lebesgue measure $m$ and by $h_{\lambda, \omega}$ its density. As a function in $\mathrm{L}^{1}(m), h_{\lambda, \omega}$ varies smoothly with the parameters.

Theorem 4.1. For any $1 \leq p<\infty$,

(1) the map $(\lambda, \omega) \mapsto h_{\lambda, \omega}$ from $(3,+\infty) \times \mathbb{R}$ to $\mathrm{L}^{p}(m)$ is continuous;

(2) for any $\lambda>3$, the map $\omega \mapsto h_{\lambda, \omega}$ from $\mathbb{R}$ to $\mathrm{L}^{p}(m)$ is $\alpha$-Hölder continuous for any exponent $0<\alpha<1$.

This is false in $\mathrm{L}^{\infty}(m)$ as explained below. Since $\rho_{\text {as }}$ is obtained by integration with respect to the measure $\mu_{\lambda, \omega}$, the same result is true for the maps $(\lambda, \omega) \mapsto$ $\rho_{\text {as }}(\lambda, \omega)$ and $\omega \mapsto \rho_{\text {as }}(\lambda, \omega)$ and proves Theorem 1.1. It will follow from Section 5 that for most $\lambda$, the map $\omega \mapsto \rho_{\text {as }}(\lambda, \omega)$ is not Lipschitz, so that the exponents given by Theorem 4.1 are optimal. The proof gives however a sharper modulus of continuity in $\omega|\log \omega|$.

Theorem 4.1 also gives the following corollary.

Corollary 4.2. For any $\lambda>3$, the set of parameters $\omega$ such that $\rho_{a s}(\lambda, \omega)$ is irrational has Hausdorff dimension equal to 1.

This is a first step towards Theorem 1.2.

Proof. A map which is $\alpha$-Hölder continuous sends any set with Hausdorff dimension $\beta$ onto a set whose Hausdorff dimension is bounded by $\beta / \alpha$. Here, $\alpha$ is arbitrarily close to 1 , and the image (the set of irrational numbers) has dimension $\beta=1$.

4.2. The symbolic Perron-Frobenius operator. We sketch here the proof of Theorem 4.1. The main idea is to introduce some operator with suitable properties.

Recall first that the absolutely continuous invariant measures can be obtained as fixed points for the Perron-Frobenius linear operator $\mathcal{L}$ in the space of the densities that have bounded variations, $\mathrm{BV}\left(\mathbb{T}^{1}\right)$ : for any absolutely continuous invariant measure $\mu=h . m$ with density $h$ in $\mathrm{BV}\left(\mathbb{T}^{1}\right)$, the measure $f(\mu)$ is also absolutely continuous with respect to the Lebesgue measure. The Perron-Frobenius operator 

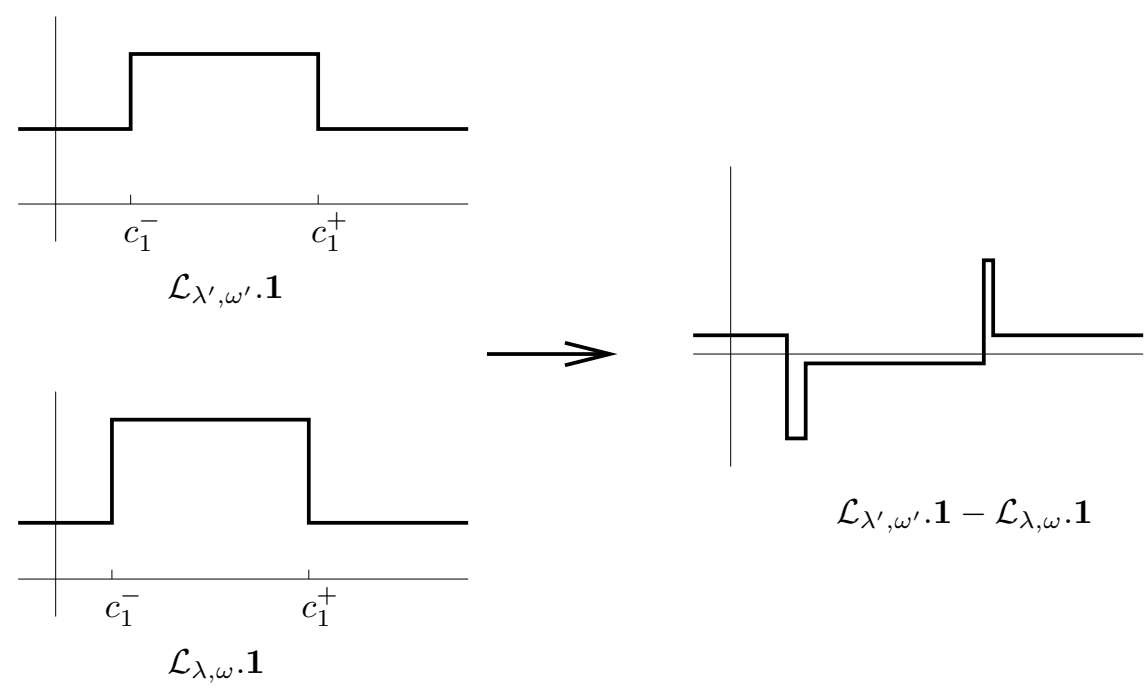

FiguRE 4. Discontinuity of $(\lambda, \omega) \mapsto \mathcal{L}_{\lambda, \omega}$.

associates to the density $h$ the density $\mathcal{L} . h$ of the measure $f(\mu)$. It is also defined by the following equality:

$$
(\mathcal{L} . h)(x)=\sum_{f(y)=x} \frac{h(y)}{|D f(y)|} .
$$

This operator has good spectral properties: in particular, the eigenvalue 1 is isolated, so that one can expect to follow the fixed points after perturbation of $\mathcal{L}$.

However, the Perron-Frobenius operator $\mathcal{L}_{\omega, \lambda}$ acting on $\mathrm{BV}\left(\mathbb{T}^{1}\right)$ and associated to the map $f_{\lambda, \omega}$ does not vary continuously with the parameters $(\lambda, \omega)$. This can be easily seen since the constant function $\mathbf{1}$ is mapped by $\mathcal{L}_{\lambda, \omega}$ onto a function which takes on two values. For closeby parameters $(\lambda, \omega)$ and $\left(\lambda^{\prime}, \omega^{\prime}\right)$, the function

$$
\mathcal{L}_{\lambda^{\prime}, \omega^{\prime} \cdot \mathbf{1}}-\mathcal{L}_{\lambda, \omega} \cdot \mathbf{1}
$$

is small in any space $\mathrm{L}^{p}(m)$ but not in $\mathrm{L}^{\infty}(m)$ or in $\mathrm{BV}\left(\mathbb{T}^{1}\right)$ (see Figure $\mathbb{1}$ ): note that the sizes and the positions of the discontinuities of the two functions are close together. But since the positions of the discontinuities are sligthly different, the difference between the two functions has large discontinuities.

This problem can be bypassed by changing the function space. Like for $\mathcal{L}_{\lambda, \omega} . \mathbf{1}$, all the functions $\mathcal{L}_{\lambda, \omega}^{n} . \mathbf{1}(n \geq 1)$ are piecewise constant with discontinuities along the orbits of the critical points $c_{0}^{+}$and $c_{0}^{-}$. This leads us to work in a sequence space: we first choose any $1<u<\lambda / 2$. For $\mathbf{s}=\left(v,\left(s_{n}^{-}\right)_{n \geq 1},\left(s_{n}^{+}\right)_{n \geq 1}\right)$ in $\mathbb{R} \times \mathbb{R}^{\mathbb{N}} \times \mathbb{R}^{\mathbb{N}}$, we then set

$$
\|\mathbf{s}\|=|v|+\sum_{n \geq 1}\left(\left|s_{n}^{-}\right|+\left|s_{n}^{+}\right|\right) u^{n}
$$

and define the Banach space

$$
\hat{E}_{u}=\left\{\mathbf{s},\|\mathbf{s}\|<\infty \text { and } \sum_{n \geq 1}\left(s_{n}^{-}+s_{n}^{+}\right)=0\right\} .
$$


Any element $\mathbf{s}$ of $\hat{E}_{u}$ can be identified by some linear map $i_{\lambda, \omega}$ to a function in $\mathrm{L}^{1}(m)$ defined by

$$
i_{\lambda, \omega} \cdot \mathbf{S}(x)=v+\int_{[0, x)} \sum_{n}\left(s_{n}^{-} \delta_{c_{n}^{-}}+s_{n}^{+} \delta_{c_{n}^{+}}\right)
$$

where $\delta_{x}$ is the Dirac measure at $x$ and $\left(c_{n}^{-}\right)$and $\left(c_{n}^{+}\right)$the postcritical orbits. (The condition $\sum_{n \geq 1}\left(s_{n}^{-}+s_{n}^{+}\right)=0$ in 4.2 ) assures the periodicity of $i_{\lambda, \omega . s .)}$

A linear Perron-Frobenius operator $\hat{\mathcal{L}}_{\lambda, \omega}$ can be defined on $\hat{E}_{u}$ (it is not unique in general) so that the following diagram commutes:

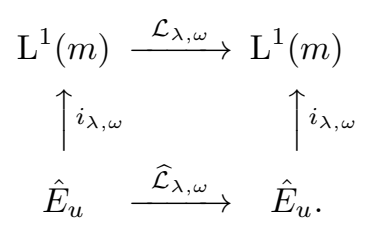

The definition of $\widehat{\mathcal{L}}_{\lambda, \omega}$ is very natural from the definition (4.1) of $\mathcal{L}_{\lambda, \omega}$ but is simpler when the following generic assumption is satisfied (we will not discuss in detail the general situation here):

$$
\forall n \geq 1, \quad c_{n}^{-}, c_{n}^{+} \notin\left\{c_{0}^{-}, c_{0}^{+}\right\} .
$$

(no collision condition)

In this case, for any $\mathbf{s}=\left(v,\left(s_{n}^{-}\right),\left(s_{n}^{+}\right)\right)$, the values of $i_{\lambda, \omega} \cdot \mathbf{s}$ at $c_{0}^{-}$and $c_{0}^{+}$are well defined and equal to

$$
v^{-}=v, \quad v^{+}=v+\sum_{c_{n}^{-} \in \pi\left(\left[0, \frac{1+\lambda}{2 \lambda}\right)\right)} s_{n}^{-}+\sum_{c_{n}^{+} \in \pi\left(\left[0, \frac{1+\lambda}{2 \lambda}\right)\right)} s_{n}^{+},
$$

respectively. Let us define $\eta_{n}^{-}=-1$ or +1 in the case when $c_{n}^{-}$belongs to the decreasing or increasing interval of $f_{\lambda, \omega}$. One defines similarly $\eta_{n}^{+}$. The image sequence $\mathbf{r}=\left(w,\left(r_{n}^{-}\right),\left(r_{n}^{+}\right)\right)=\widehat{\mathcal{L}}_{\lambda, \omega} \cdot \mathbf{s}$ is defined by

$$
r_{1}^{-}=\frac{2 v^{-}}{\lambda}, \quad r_{1}^{+}=\frac{-2 v^{+}}{\lambda}, \quad \text { for } n \geq 2, \quad r_{n}^{ \pm}=\frac{\eta_{n-1}^{ \pm} s_{n-1}^{ \pm}}{\lambda}
$$

and the value of $w$ may now be computed by noting that the integrals of $i_{\lambda, \omega}$.s and $i_{\lambda, \omega} . \mathbf{r}$ are equal.

We introduced this space $\hat{E}_{u}$ because the operator $\widehat{\mathcal{L}}_{\lambda, \omega}$ can be shown to have both of the following properties:

Spectral property: The operator $\widehat{\mathcal{L}}_{\lambda, \omega}$ keeps the good spectral properties of the initial operator $\mathcal{L}_{\lambda, \omega}$ : the eigenvalue 1 is isolated and has multiplicity 1 . This is a very classical argument if one shows that the essential spectral radius of $\widehat{\mathcal{L}}_{\lambda, \omega}$ is strictly less than 1 . The idea is to consider the projection $\mathcal{P}: \hat{E}_{u} \rightarrow \hat{E}_{u}$ defined by

$$
\mathcal{P}\left(v,\left(s_{n}^{-}\right),\left(s_{n}^{+}\right)\right)=\left(v,\left(s_{1}^{-}, 0,0, \ldots\right),\left(s_{1}^{+}, 0,0, \ldots\right)\right) .
$$

Note that $\mathcal{P} \circ \widehat{\mathcal{L}}_{\lambda, \omega}$ is a finite rank operator and from $(4.4$ the norm of $\widehat{\mathcal{L}}_{\lambda, \omega}-\mathcal{P} \circ \widehat{\mathcal{L}}_{\lambda, \omega}$ is bounded by $\frac{u}{\lambda}$. Consequently (see DS lemma VIII.8.2), the spectrum of $\widehat{\mathcal{L}}_{\lambda, \omega}$ outisde the disk $D\left(0, \frac{u}{\lambda}\right)$ consists of a finite set of eigenvalues. 
Continuity property: The eigenspace associated to the eigenvalue 1 of the operator $\widehat{\mathcal{L}}_{\lambda, \omega}$ varies continuously with the dynamics $f_{\lambda, \omega}$. This comes from the fact that, for each density, the space $\hat{E}_{u}$ codes each discontinuity by its size but forgets its position on the circle.

In particular, the obstruction to the continuity in the space $\mathrm{BV}\left(\mathbb{T}^{1}\right)$ is no longer true: this can be seen on the example given before by Figure 4 : the image of $\mathbf{1}$ by $\widehat{\mathcal{L}}_{\lambda, \omega}$ is

$$
\left(1+\left(\|\omega\|-\left\|\frac{1+\lambda}{2}+\omega\right\|\right) \frac{2}{\lambda},\left(\frac{2}{\lambda}, 0,0, \ldots\right),\left(\frac{-2}{\lambda}, 0,0, \ldots\right)\right),
$$

where $\|x\|$ denotes the fractional part of $x$. Note that $c_{1}^{-}$and $c_{1}^{+}$are equal to $\pi(\omega)$ and $\pi\left(\frac{1+\lambda}{2}+\omega\right)$. When the "no collision condition" is satisfied, $c_{1}^{-}$ and $c_{1}^{+}$are distinct from $c_{0}^{-}$so that $\omega$ and $\frac{1+\lambda}{2}+\omega$ have their integer parts locally constant (and their fractional parts vary locally continuously). The continuity of $\widehat{\mathcal{L}}_{\lambda, \omega} . \mathbf{1}$ with $(\lambda, \omega)$ is then clear.

One may obtain the continuity of the eigenspace associated to 1 by noting that for any eigenvector $\mathbf{s}=\left(v,\left(s_{n}^{-}\right),\left(s_{n}^{+}\right)\right)$, the sequences $\left(s_{n}^{-}\right)$and $\left(s_{n}^{+}\right)$decrease like $\left(\lambda^{-n}\right)$ and that $\lambda^{-1}<u^{-1}$. Consequently, the continuity of the eigenspace with respect to the parameters $(\lambda, \omega)$ will follow from the continuity of the projection of $\widehat{\mathcal{L}}_{\lambda, \omega}$ on each coordinate of $\mathbb{R} \times \mathbb{R}^{\mathbb{N}} \times \mathbb{R}^{\mathbb{N}}$. This is a consequence of the above definition of the operator after noting that for nearby parameters that satisfy the no collision condition, each sequence $\left(\eta_{n}^{-}\right)$or $\left(\eta_{n}^{+}\right)$coincides up to an arbitrarily large integer $n$.

This idea to introduce an operator acting on a symbolic space is inspired by [T00].

4.3. Conclusion of the proof of Theorem 4.1. This time and thanks to the above properties, the argument announced at the beginning of the previous section can be carried out: the eigenspace associated to the eigenvalue 1 can be followed continuously in the space $\hat{E}_{u}$ when the parameters $(\lambda, \omega)$ vary. It is then easy to show that the image by $i_{\lambda, \omega}$ also varies continuously in $\mathrm{L}^{1}(m)$ : let $\mathbf{s}=\left(v,\left(s_{n}^{-}\right),\left(s_{n}^{+}\right)\right)$ and $\mathbf{r}=\left(w,\left(r_{n}^{-}\right),\left(r_{n}^{+}\right)\right)$be the two fixed points of closeby operators $\widehat{\mathcal{L}}_{\lambda, \omega}$ and $\widehat{\mathcal{L}}_{\lambda^{\prime}, \omega^{\prime}}$ that code for some probability densities $h$ and $h^{\prime}$. Let us compute the distance between $h$ and $h^{\prime}$ in $\mathrm{L}^{1}$. From (4.3), we get:

$$
\begin{aligned}
& \int\left|h-h^{\prime}\right| \mathrm{d} m \leq|v-w|+\sum_{n \geq 1}\left(\left|s_{n}^{-}-r_{n}^{-}\right|+\left|s_{n}^{+}-r_{n}^{+}\right|\right)+ \\
& +\sum_{n \geq 1}\left(\left|s_{n}^{-}\right|+\left|r_{n}^{-}\right|\right) \mathrm{d}\left(c_{n}^{-}(\lambda, \omega), c_{n}^{-}\left(\lambda^{\prime}, \omega^{\prime}\right)\right)+\sum_{n \geq 1}\left(\left|s_{n}^{+}\right|+\left|r_{n}^{+}\right|\right) \mathrm{d}\left(c_{n}^{+}(\lambda, \omega), c_{n}^{+}\left(\lambda^{\prime}, \omega^{\prime}\right)\right) .
\end{aligned}
$$

Note that the sequences $\left(s_{n}^{-}\right),\left(s_{n}^{+}\right),\left(r_{n}^{-}\right)$and $\left(r_{n}^{+}\right)$decrease geometrically as $\lambda^{-n}$ or $\lambda^{\prime-n}$ (see (4.4)). Let us consider some large integer $N$ in order to truncate the sums:

$$
\begin{aligned}
& \int\left|h-h^{\prime}\right| \mathrm{d} m \leq\|\mathbf{s}-\mathbf{r}\|+\frac{2|v| \lambda^{-N}}{\lambda-1}+\frac{2|w| \lambda^{\prime-N}}{\lambda^{\prime}-1}+ \\
& 2(|v|+|w|) \sum_{n=1}^{N}\left(\mathrm{~d}\left(c_{n}^{-}(\lambda, \omega), c_{n}^{-}\left(\lambda^{\prime}, \omega^{\prime}\right)\right)+\mathrm{d}\left(c_{n}^{-}(\lambda, \omega), c_{n}^{-}\left(\lambda^{\prime}, \omega^{\prime}\right)\right)\right) .
\end{aligned}
$$

By taking $N$ large enough and the parameters $(\lambda, \omega)$ and $\left(\lambda^{\prime}, \omega^{\prime}\right)$ close, one gets that $h-h^{\prime}$ is arbitrarily small in $\mathrm{L}^{1}$ and proves at last the continuity of $h$ in $\mathrm{L}^{1}$ 
with respect to the parameters. Some improvements of these arguments yield the other conclusions of Theorem 4.1.

\section{Generic values}

5.1. Main results. The approach of this section is independent of the previous one. All the results stated here are consequences of the following technical theorem:

Theorem 5.1. There exist a constant $C_{0}>0$, a slope $\lambda_{0}>3$ and some subset $E$ of the parameter space $\left(\lambda_{0},+\infty\right) \times \mathbb{R}$ with full Lebesgue measure such that for any $\left(\lambda, \omega_{0}\right) \in E$ and $K>0$, there exist $0<r<K^{-1}$ and $P>K$ satisfying

$$
\sup _{\left|\omega-\omega_{0}\right|<r}\left|\rho_{a s}(\lambda, \omega)-\left(\rho_{a s}\left(\lambda, \omega_{0}\right)+P .\left(\omega-\omega_{0}\right)\right)\right|<C_{0} . r .
$$

In other words, for any $\left(\lambda, \omega_{0}\right)$, the slope of the map $\omega \mapsto \rho_{\text {as }}(\lambda, \omega)$ at $\omega_{0}$ is arbitrarily large at some scales. The proof shows that at those points the slope may be negative or positive depending on the scale $r$. Thus, for most $\lambda$ the map $\omega \mapsto \rho_{\text {as }}(\lambda, \omega)$ is not so smooth. In particular, we get the following corollary.

Corollary 5.2. There exists a slope $\lambda_{0}$ such that for Lebesgue-almost any slope $\lambda>\lambda_{0}$, the map $\omega \mapsto \rho_{a s}(\lambda, \omega)$ is not differentiable on a full Lebesgue measure set. Hence, it is not absolutely continuous.

It is not true for all values of $\lambda$ since for $\lambda=2 n+1$ with $n \in \mathbb{N} \backslash\{0\}$, the usual Lebesgue measure is preserved so that $\rho_{\text {as }}(\lambda, \omega)=\omega+C$, where $C$ depends only on $\lambda$.

Theorem 5.1 also has a positive consequence, the following corollary, which proves Theorem 1.2:

Corollary 5.3. There exists a slope $\lambda_{0}>3$ such that for any $\rho_{0} \in \mathbb{R}$, the set of parameters $\left(\left(\lambda_{0},+\infty\right) \times \mathbb{R}\right) \cap \rho^{-1}\left(\rho_{0}\right)$ has zero Lebesgue measure.

Proof. Let $E$ be the set given by Theorem 5.1. One argues by contradiction: if $\left(\left(\lambda_{0},+\infty\right) \times \mathbb{R}\right) \cap \rho^{-1}\left(\rho_{0}\right)$ has positive measure, there exist $\lambda>\lambda_{0}$ and $\omega_{0} \in \mathbb{R}$ such that $\left(\lambda, \omega_{0}\right)$ belongs to $E$ and is a density point of the set $(\{\lambda\} \times \mathbb{R}) \cap \rho^{-1}\left(\rho_{0}\right)$ in $\{\lambda\} \times \mathbb{R}$ for the Lebesgue measure.

By Theorem 5.1 with constant $K=3 C_{0}$, there exists a sequence $\left(r_{n}\right)$ of positive real numbers that converges to 0 such that for any $\omega$ with $\frac{1}{2} r_{n}<\left|\omega-\omega_{0}\right|<r_{n}$,

$$
\left|\rho_{\text {as }}(\lambda, \omega)-\rho_{\text {as }}\left(\lambda, \omega_{0}\right)\right|>K\left|\omega-\omega_{0}\right|-C_{0} r_{n}>\left(\frac{K}{2}-C_{0}\right) r_{n}=\frac{C_{0}}{2} r_{n},
$$

so that $\rho_{\text {as }}(\lambda, \omega) \neq \rho_{\text {as }}\left(\lambda, \omega_{0}\right)$. The density of $(\{\lambda\} \times \mathbb{R}) \cap \rho^{-1}\left(\rho_{0}\right)$ in $\{\lambda\} \times\left(\omega_{0}-\right.$ $\left.r_{n}, \omega_{0}+r_{n}\right)$ is smaller than $\frac{1}{2}$, which is impossible since $\left(\lambda, \omega_{0}\right)$ is a density point of $(\{\lambda\} \times \mathbb{R}) \cap \rho^{-1}\left(\rho_{0}\right)$.

5.2. Expression of $\rho_{\text {as }}$. We now sketch the proof of Theorem 5.1. The first step shows that the almost sure rotation number may be approximated by some affine functions of $\omega$, at some scales of the parameter space (see Lemma 5.5).

The dynamics is controlled by the critical orbits $\left(c_{n}^{-}\right)$and $\left(c_{n}^{+}\right)$. Let us recall that we defined $\eta_{n}^{-}=-1$ or +1 in the case when $c_{n}^{-}$belongs to the decreasing or increasing interval of $f_{\lambda, \omega}$. We defined similarly $\eta_{n}^{+}$. The sequences $\left(\eta_{n}^{-}\right)$and $\left(\eta_{n}^{+}\right)$ 
are called the kneading sequences of the dynamics. It is also useful to define two derived sequences $\left(\varepsilon_{n}^{-}\right)$and $\left(\varepsilon_{n}^{+}\right)$by

$$
\varepsilon_{1}^{-}=1, \quad \varepsilon_{1}^{+}=-1, \text { and for any } n \geq 1, \varepsilon_{n+1}^{ \pm}=\eta_{n}^{ \pm} \varepsilon_{n}^{ \pm} .
$$

They correspond to the sign of the jumps $\left(s_{n}^{-}\right)$and $\left(s_{n}^{+}\right)$(see formulas (4.4)).

The position of $c_{n}^{-}$is coded by its lift $l_{n}^{-}$in $\left(\frac{-\lambda+1}{2 \lambda}, \frac{\lambda+1}{2 \lambda}\right]$. One introduces in the same way the position $l_{n}^{+}$of $c_{n}^{+}$.

The density $h_{\lambda, \omega}$ of the measure $\mu_{\lambda, \omega}$ may now be computed explicitly using the slope $\lambda$, the kneading invariants $\left(\eta_{n}^{-}\right)$and $\left(\eta_{n}^{+}\right)$and the positions $\left(l_{n}^{-}\right)$and $\left(l_{n}^{+}\right)$.

This gives the following formula for the almost sure rotation number.

\section{Lemma 5.4.}

where

$$
\rho_{a s}-\omega=\frac{A}{B}
$$

$$
A=\frac{\frac{\lambda^{2}-1}{4 \lambda}-\sum_{n \geq 1} \eta_{n}^{+} \varepsilon_{n}^{+}\left(\lambda-\eta_{n}^{+}\right) \lambda^{-n}\left(l_{n}^{+}\right)^{2}}{1-\sum_{\eta_{n}^{+}=1} 2 \varepsilon_{n}^{+} \lambda^{-n}}+\frac{-\sum_{n \geq 1} \eta_{n}^{-} \varepsilon_{n}^{-}\left(\lambda-\eta_{n}^{-}\right) \lambda^{-n}\left(l_{n}^{-}\right)^{2}}{1+\sum_{\eta_{n}^{-}=1} 2 \varepsilon_{n}^{-} \lambda^{-n}}
$$

and

$$
B=\frac{1-\sum_{n \geq 1} 2 \varepsilon_{n}^{+} \lambda^{-n} l_{n}^{+}}{1-\sum_{\eta_{n}^{+}=1} 2 \varepsilon_{n}^{+} \lambda^{-n}}+\frac{-\sum_{n \geq 1} 2 \varepsilon_{n}^{-} \lambda^{-n} l_{n}^{-}}{1+\sum_{\eta_{n}^{-}=1} 2 \varepsilon_{n}^{-} \lambda^{-n}} .
$$

Proof. We give the main steps of the proof and omit the computations.

Let $\mathbf{s}=\left(v,\left(s_{n}^{-}\right),\left(s_{n}^{+}\right)\right)$be the element of $\hat{E}_{u}$ that codes for the density $h_{\lambda, \omega}$. As in (4.4), we introduce the values $v^{-}$and $v^{+}$of $h_{\lambda, \omega}$ at $c_{0}^{-}$and $c_{0}^{+}$. In particular:

$$
v^{-}=v \quad \text { and } \quad v^{+}=v^{-}+\sum_{\eta_{n}^{-}=1} s_{n}^{-}+\sum_{\eta_{n}^{+}=1} s_{n}^{+} .
$$

The rotation number $\rho_{\text {as }}$ was defined as $\int_{[0,1)}\left(\tilde{f}_{\lambda, \omega}-\mathrm{Id}\right) h_{\lambda, \omega} \mathrm{d} m$. This gives

$$
\rho_{\text {as }}-\omega=\frac{\lambda^{2}-1}{4 \lambda} v^{+}-\sum_{n \geq 1} \eta_{n}^{-} \frac{s_{n}^{-}}{2}\left(\lambda-\eta_{n}^{-}\right) l_{n}^{-2}-\sum_{n \geq 1} \eta_{n}^{+} \frac{s_{n}^{+}}{2}\left(\lambda-\eta_{n}^{+}\right) l_{n}^{+^{2}} .
$$

We first recall that $h_{\lambda, \omega}$ is normalized so that the measure $h_{\lambda, \omega} . m$ is a probability. We get:

$$
v^{+}-\sum_{n \geq 1}\left(s_{n}^{-} l_{n}^{-}+s_{n}^{+} l_{n}^{+}\right)=1
$$

The invariance of $h_{\lambda, \omega}$ by $\mathcal{L}_{\lambda, \omega}$ gives for $n \geq 1$,

$$
s_{n}^{-}=\frac{2 v^{-} \varepsilon_{n}^{-}}{\lambda^{n}}, \quad s_{n}^{+}=\frac{2 v^{+} \varepsilon_{n}^{+}}{\lambda^{n}} .
$$

This allows us to eliminate $\left(s_{n}^{-}\right)$and $\left(s_{n}^{+}\right)$in formulas (5.1) and (5.3). Using those two equalities, one can express $v^{+}$with the slope $\lambda$, the kneading invariants $\left(\eta_{n}^{-}\right)$ and $\left(\eta_{n}^{+}\right)$and the positions $\left(l_{n}^{-}\right)$and $\left(l_{n}^{+}\right)$.

From these relations, one supresses in (5.2) the occurrences of $v^{+},\left(s_{n}^{-}\right)$and $\left(s_{n}^{+}\right)$and obtains the desired equality. 
5.3. Approximation of $\rho_{\text {as }}$. Let us fix some slope $\lambda>3$. One can approximate the density $h_{\lambda, \omega}$ and the almost sure rotation number by truncating the terms which depend on the quantities $\eta_{k}^{-}, \eta_{k}^{+}, l_{k}^{-}$and $l_{k}^{+}$with order $k$ larger than $n$. One sees that some discontinuities in the truncated sums appear when the signs of $\eta_{k}^{ \pm}$ for $1 \leq k \leq n$ change. This led us to introduce the following definition (which appeared already in Section 4.2 as the no collision condition):

We will say that no collision with order smaller than $n \geq 1$ occurs on some interval $\left(\omega_{1}, \omega_{2}\right)$ if for any $\omega \in\left(\omega_{1}, \omega_{2}\right)$ and any $1 \leq k \leq n$,

$$
\left\{c_{k}^{-}, c_{k}^{+}\right\} \cap\left\{c_{0}^{-}, c_{0}^{+}\right\}=\emptyset .
$$

The truncated sums of order $n$ thus vary continuously as long as no collision with order smaller than $n \geq 1$ occur.

A sharper approximation gives the following estimates on the variations of $\omega \mapsto \rho_{\text {as }}(\lambda, \omega)$ : on some intervals of the parameter space $\omega$, the number $\rho_{\text {as }}$ is approached by some affine function whose slope $\Delta_{n} \rho_{\text {as }}$ is essentially equal to

$$
\sum_{1 \leq k \leq n}\left(\eta_{k}^{+}\left(\lambda-\eta_{k}^{+}\right) l_{k}^{+}-\eta_{k}^{-}\left(\lambda-\eta_{k}^{-}\right) l_{k}^{-}\right) .
$$

More precisely:

Lemma 5.5 (Approximation of $\rho_{\mathrm{as}}$ ). There exist some universal constant $C_{1}$, and some function $(\lambda, \omega) \mapsto B(\lambda, \omega)$ bounded from below and above with the following property: For any slope $\lambda>3$, let us consider $n \geq 1$ and some interval $\left(\omega_{1}, \omega_{2}\right)$ with no collision of order smaller than $n$. Then,

$$
\left|\rho_{a s}\left(\lambda, \omega_{2}\right)-\rho_{a s}\left(\lambda, \omega_{1}\right)-\Delta_{n} \rho_{a s}\left(\lambda, \omega_{1}\right) \cdot\left(\omega_{2}-\omega_{1}\right)\right|<C_{1} \lambda^{-n+1},
$$

where

$$
\Delta_{n} \rho_{a s}(\lambda, \omega)=1+\lambda^{-1} B(\lambda, \omega) \sum_{1 \leq k \leq n}\left(\eta_{k}^{+}\left(\lambda-\eta_{k}^{+}\right) l_{k}^{+}-\eta_{k}^{-}\left(\lambda-\eta_{k}^{-}\right) l_{k}^{-}\right) .
$$

Proof. In order to explain how this estimate can be proven, we consider the expression of $\rho_{\text {as }}$ given by Lemma 5.4. Let us note that $A$ is close to $\frac{\lambda}{4}$ and $B$ close to 1 . Hence, one can roughly approximate $\rho_{\text {as }}\left(\lambda, \omega_{2}\right)-\rho_{\text {as }}\left(\lambda, \omega_{1}\right)-\left(\omega_{2}-\omega_{1}\right)$ by

$$
\left(A\left(\lambda, \omega_{2}\right)-A\left(\lambda, \omega_{1}\right)\right)-\frac{\lambda}{4}\left(B\left(\lambda, \omega_{2}\right)-B\left(\lambda, \omega_{1}\right)\right) .
$$

The expected estimate allows an error of order $\lambda^{-n+1}$. Hence, one can truncate in the expressions for $A$ and $B$ the terms with order larger than $n$.

An easy computation shows that

$$
\varepsilon_{k}^{-} \frac{\partial}{\partial \omega} c_{k}^{-} \simeq \lambda^{k-1}, \quad \varepsilon_{k}^{+} \frac{\partial}{\partial \omega} c_{k}^{+} \simeq-\lambda^{k-1} .
$$

Let us consider in particular the points $c_{n}^{ \pm}$. As no collision of order $n$ occurs on $\left(\omega_{1}, \omega_{2}\right)$, we have by (5.7),

$$
\left|\omega_{2}-\omega_{1}\right|<2 \lambda^{-n+1} .
$$

Since no collision of order smaller than $n$ occurs on $\left(\omega_{1}, \omega_{2}\right)$, the quantities $\eta_{k}^{ \pm}$ and $\varepsilon_{k}^{ \pm}$, with $1 \leq k \leq n$, are constant on this interval. The length $l_{k}^{ \pm}$varies like $\frac{\partial c_{k}^{ \pm}}{\partial \omega}$. One deduces by (5.7) that the term $\left|B\left(\lambda, \omega_{2}\right)-B\left(\lambda, \omega_{1}\right)\right|$ may be bounded (up to a constant) by $n \lambda^{-1}\left|\omega_{2}-\omega_{1}\right|$. A finer (but straightforward) computation 
shows that one has in fact the bound $\lambda^{-2}\left|\omega_{2}-\omega_{1}\right|$. Hence from (5.8) the variations of $\lambda . B$ are bounded by $\lambda^{-n}$ and may be neglected.

We now come to the term $A\left(\lambda, \omega_{2}\right)-A\left(\lambda, \omega_{1}\right)$. Here the dependence in $l_{k}^{ \pm}$is quadratic. First, the variation of $A$ can be estimated (up to some bounded factor) by:

$$
\left(-\sum_{1 \leq k \leq n} \eta_{k}^{+} \varepsilon_{k}^{+}\left(\lambda-\eta_{k}^{+}\right) \lambda^{-k} 2 l_{k}^{+} \frac{\partial c_{k}^{+}}{\partial \omega}-\sum_{1 \leq k \leq n} \eta_{k}^{-} \varepsilon_{k}^{-}\left(\lambda-\eta_{k}^{-}\right) \lambda^{-k} 2 l_{k}^{-} \frac{\partial c_{k}^{-}}{\partial \omega}\right) \cdot\left(\omega_{2}-\omega_{1}\right)
$$

and from (5.7) this may be approached by:

$$
2 \lambda^{-1}\left(\sum_{1 \leq k \leq n} \eta_{k}^{+}\left(\lambda-\eta_{k}^{+}\right) l_{k}^{+}-\sum_{1 \leq k \leq n} \eta_{k}^{-}\left(\lambda-\eta_{k}^{-}\right) l_{k}^{-}\right) \cdot\left(\omega_{2}-\omega_{1}\right)
$$

which shows how the sum (5.5) appears. These estimates together imply the lemma.

5.4. Localisation of the critical orbits. We now come to the second step of the proof of Theorem 5.1.

In order to control the sum (5.5), it is convenient to estimate the positions $l_{k}^{-}$and $l_{k}^{+}$of the critical images $c_{k}^{-}$and $c_{k}^{+}$for $1 \leq k \leq n$. The following result describes the behaviour of the critical orbits for any $\omega$ in some interval of length $\simeq \lambda^{-n}$ : first, it asserts that during $p$ iterates the critical images $c_{k}^{-}$and $c_{k}^{+}$belong to some prescribed regions of the circle (namely equal to $\pi\left(\left(0, \frac{1}{8}\right)\right)$ and $\pi\left(\left(\frac{3}{16}, \frac{5}{16}\right)\right)$ respectively); second it shows that no collision with order smaller than $n$ occurs on this interval (so that Lemma 5.5 can be applied). Note that this second property in the statement of Theorem 5.6 allows us to choose the integer $n$ in some interval $[m, m+p]$ of length $p$. Theorem 5.1 will then be a direct consequence of Theorem 5.6 and of Lemma 5.5.

Theorem 5.6 (Localisation of the critical orbits). There exist some constants $C_{2}>0$ and $\lambda_{0}>3$ and some full Lebesgue measure subset $E$ of $\left(\lambda_{0},+\infty\right) \times \mathbb{R}$ which satisfies the following property: for any $(\lambda, \omega) \in E$ and $p \geq 1$, there exists $m \geq 1$ such that:

(1) $l_{m}^{-}, \ldots, l_{m+p}^{-} \in\left(0, \frac{1}{8}\right)$ and $l_{m}^{+}, \ldots, l_{m+p}^{+} \in\left(\frac{3}{16}, \frac{5}{16}\right)$;

(2) for any $m \leq n \leq m+p$, there is no collision of order smaller than $n$ on $\left(\omega-C_{2} \lambda^{-n+1}, \omega+C_{2} \lambda^{-n+1}\right)$.

5.5. Parameters exclusion: proof of Theorem 5.6. This part of the proof is long and technical. However the arguments are similar to a standard situation in one-dimensional dynamics: the parameters exclusion, that first appeared in [J].

Let us recall the estimate $(5.7)$ :

$$
\left|\frac{\partial}{\partial \omega} c_{k}^{-}\right|,\left|\frac{\partial}{\partial \omega} c_{k}^{+}\right| \simeq \lambda^{k-1}
$$

We consider some small neighborhoods $V^{-}$and $V^{+}$of $\pi(1 / 16)$ and $\pi(1 / 4)$ respectively. In order to prove Theorem 5.6, we look for parameters $\left(\lambda, \omega_{0}\right)$ such that for any $p \geq 1$, there exists $m \geq 1$ such that:

(1) $c_{m}^{-}, \ldots, c_{m+p}^{-} \in V^{-}$and $c_{m}^{+}, \ldots, c_{m+p}^{+} \in V^{+}$. 
(2) For any $1 \leq k \leq m-1$,

$$
\mathrm{d}\left(c_{m-k}^{-},\left\{c_{0}^{-}, c_{0}^{+}\right\}\right), \mathrm{d}\left(c_{m-k}^{+},\left\{c_{0}^{-}, c_{0}^{+}\right\}\right)>\lambda^{-k} / 4 .
$$

Theorem 5.6(便) is a direct consequence of (11). Theorem 5.6(2) is obtained from both (11) and (2) with the estimate (5.9). More precisely, the estimate (2) is given for the initial parameter $\omega_{0}$. If $\omega$ is close to $\omega_{0}$, we now obtain for any $1 \leq k \leq m-1$,

$$
d\left(\left\{c_{m-k}^{-}, c_{m-k}^{+}\right\},\left\{c_{0}^{-}, c_{0}^{+}\right\}\right)>\frac{\lambda^{-k}}{4}-C_{3} \lambda^{m-k-1}\left|\omega-\omega_{0}\right|,
$$

with some constant $C_{3}>0$. This is non-zero if $\left|\omega-\omega_{0}\right|<\lambda^{-m+1} / 4 C_{3}$. Thus, no collision of order smaller than $m-1$ can occur on $\left(\omega_{0}-\lambda^{-m+1} / 4 C_{3}, \omega_{0}+\lambda^{-m+1} / 4 C_{3}\right)$. Using (1), the same argument for $m \leq k \leq m+p$ implies Theorem 5.6(2).

Hence, the main step of the proof shows that if one removes a zero Lebesgue measure set, the remaining parameters satisfy both (11) and (2). This is very similar to the parameters exclusions that appeared in the proof of Jakobson's Theorem (see Section 3.3), where the parameters that have too strong recurrence near the critical set have to be avoided. Since the maps $f_{\lambda, \omega_{0}}$ here are piecewise linear, the distortion estimates are simplified.

However, the behaviour of both critical orbits can not be controlled independently since one requires returns for $\left(c_{k}^{-}\right)$and $\left(c_{k}^{+}\right)$at the same time in $V^{-}$and $V^{+}$respectively. Contrary to Jakobson's theorem, we thus need to work in the two-parameters space $(3,+\infty) \times \mathbb{R}$.

5.6. Conclusion of the proof of Theorem 5.1. Let us consider the set of parameters $E$ given by Theorem 5.6. For $\left(\lambda, \omega_{0}\right) \in E$ and $K>0$, we choose any large integer $p \gg K$. In view of the statement of Theorem 5.6, we get some integer $m$ sufficiently large so that $C_{2} \lambda^{-m+1}<K^{-1}$.

For any $m \leq n \leq m+p$, by Theorem 5.6(1) there is no collision of order smaller than $n$ on $\left(\omega_{1}, \omega_{2}\right)=\left(\omega_{0}-r_{n}, \omega_{0}+r_{n}\right)$, where $r_{n}=C_{2} \lambda^{-n+1}$. Hence, by Lemma 5.5, it is sufficient to fix some $n$ such that $\left|\Delta_{n} \rho_{\text {as }}\left(\lambda, \omega_{0}\right)\right|>K$ and set $r=r_{n}$.

Let us compare $\Delta_{m} \rho_{\text {as }}\left(\lambda, \omega_{0}\right)$ and $\Delta_{m+p} \rho_{\text {as }}\left(\lambda, \omega_{0}\right)$. By Theorem 5.6(2), each term of order $k \in\{m, \ldots, m+p\}$ in the sum (5.5) is bounded from above by $-\frac{1}{16}(\lambda-1)$ (note also that $\left.\eta_{k}^{-}=\eta_{k}^{+}=1\right)$. Hence, we get by (5.6),

$$
\Delta_{m+p} \rho_{\text {as }}\left(\lambda, \omega_{0}\right)<\Delta_{m} \rho_{\text {as }}\left(\lambda, \omega_{0}\right)-\frac{p(\lambda-1) B\left(\lambda, \omega_{0}\right)}{16 \lambda}<\Delta_{m} \rho_{\text {as }}\left(\lambda, \omega_{0}\right)-2 K .
$$

One deduces that either $\Delta_{m} \rho_{\text {as }}\left(\lambda, \omega_{0}\right)>K$ or $\Delta_{m+p} \rho_{\text {as }}\left(\lambda, \omega_{0}\right)<-K$. Consequently, $n$ may be chosen equal to one of the integers $m$ or $m+p$, which concludes the proof.

\section{References}

[A] V. ARNOL'D, Small denominators I : Mapping the circle onto itself, Izv. Akad. Nauk. SSSR Ser. Mat. 25 (1961), 21-86. See also : Translations Amer. Math. Soc. $2^{\text {nd }}$ series 46 (1965), 213-284.

[B] P. BOYLAND, Bifurcations of circle maps: Arnol'd tongues, bistability and rotation intervals, Commun. Math. Phys. 106 (1986), 353-381.

[CGT] A. CHENCINER, J.-M. GAMBAUDO, C. TRESSER, Une remarque sur la structure des endomorphismes de degré 1 du cercle, C. R. Acad. Sci. Paris Série I 299 (1984), 145-147.

[Cr01a] S. CROVISIER, Nombre de rotation presque sûr des endomorphismes du cercle affines par morceaux, Bull. Braz. Math. Soc. (N.S.) 33 (2002), 1-26. 
[Cr01b] S. CROVISIER, Nombre de rotation et dynamique faiblement hyperbolique, thèse de doctorat de l'université Paris-Sud (2001), Orsay. Available at http://tel.ccsd.cnrs.fr/documents/archives0/00/00/11/85/index_fr.html

[DS] N. DUNFORD, J. SCHWARTZ, Linear Operators, part I, John Wiley \& sons (1958).

[GS] É. GHYS, V. SERGIESCU, Sur un groupe remarquable de difféomorphismes du cercle, Comment. Math. Helv. 62 (1987), 185-239.

[G93] J. GRACZYK, Linearizable circle diffeomorphisms in one-parameter families, Bol. Soc. Bras. Mat. (N.S.) 24 (1993), 201-210.

[GŚ] J. GRACZYK, G. ŚWIA̧TEK, Critical circle maps near bifurcation, Commun. Math. Phys. 176 (1996), 227-260.

[H77] M. HERMAN, Mesure de Lebesgue et nombre de rotation, in Geometry and Topology, Lecture Notes in Mathematics 597, Springer-Verlag, Berlin (1977), 271-293.

[H79] M. HERMAN, Sur la conjugaison différentiable des difféomorphismes du cercle à des rotations, Publ. Math. Inst. Hautes Études Sci. 49 (1979), 5-233.

$[\mathrm{J}]$ M. JAKOBSON, Absolutely continuous invariant measures for one-parameter families of one-dimensional maps, Commun. Math. Phys. 81 (1981), 39-88.

[LY] A. LASOTA, J. YORKE, On the existence of invariant measures for piecewise monotonic transformations, Trans. Amer. Math. Soc. 186 (1973), 481-488.

[L] F. LEDRAPPIER, Some properties of absolutely continuous invariant measures on an interval, Ergod. Th. \& Dynam. Sys. 1 (1981), 77-93.

[Ś88] G. ŚWIA̧TEK, Rational rotation numbers for maps of the circle, Commun. Math. Phys. 119 (1988), 109-128.

[Ś89] G. ŚWIA̧TEK, Endpoints of rotation intervals for maps of the circle, Ergod. Th. \& Dynam. Sys. 9 (1989), 173-190.

[T92] M. TSUJII, Rotation number and one-parameter families of circle diffeomorphisms, Ergod. Th. \& Dynam. Sys. 12 (1992), 359-363.

[T93] M. TSUJII, Positive Lyapunov exponents in families of one-dimensional dynamical systems, Invent. Math. 111 (1993), 113-137.

[T00] M. TSUJII, A simple proof for monotonicity of entropy in the quadratic family, Ergod. Th. \& Dynam. Sys. 20 (2000), 925-933.

[WY] Q. WANG, L.-S. YOUNG, Strange attractors with one direction of unstability, Commun. Math. Phys. 218 (2001), 1-92.

Institut de Mathématiques de Bourgogne, UMR 5584, Université de Bourgogne, 9, avenue Alain savary, B.P. 47 870, F-21078 Dijon Cedex France.

E-mail address: Sylvain.Crovisier@u-bourgogne.fr 